\title{
On a q-analog of a Sahi result
}

\author{
Olga Bershtein \\ Institute for Low Temperature Physics and Engineering, Kharkov, Ukraine \\ e-mail: bershtein@ilt.kharkov.ua
}

\begin{abstract}
We obtain a $q$-analog of a well known Sahi result on the joint spectrum of $S\left(G L_{n} \times G L_{n}\right)$-invariant differential operators with polynomial coefficients on the vector space of complex $n \times n$-matrices.

Keywords: factorial Schur polynomials, Capelli identities, quantum groups, quantum prehomogeneous vector spaces.

MSC: 17B37, 20G42, 16S32.
\end{abstract}

\section{Introduction}

Start with recalling some well-known facts. Denote by Mat ${ }_{n}$ the vector space of complex $n \times n$ matrices. The group $K=S\left(G L_{n} \times G L_{n}\right)$ acts on Mat $_{n}$ by

$$
(u, v) Z=u Z v^{-1}, \quad(u, v) \in K, Z \in \mathrm{Mat}_{n}
$$

This induces the natural $K$-actions in the vector spaces $\mathbb{C}\left[\mathrm{Mat}_{n}\right]$ of holomorphic polynomials, D $\left[\mathrm{Mat}_{n}\right]$ of differential operators with constant coefficients, and PD[Mat $\left.{ }_{n}\right]$ of differential operators with polynomial coefficients. The well-known Hua theorem claims that

$$
\mathbb{C}\left[\mathrm{Mat}_{n}\right]=\bigoplus_{\lambda \in \Lambda_{n}} \mathbb{C}\left[\mathrm{Mat}_{n}\right]_{\lambda}
$$

where $\Lambda_{n}=\left\{\lambda=\left(\lambda_{1}, \lambda_{2}, \ldots, \lambda_{n}\right) \in \mathbb{Z}_{+}^{n} \mid \lambda_{1} \geq \lambda_{2} \geq \ldots \geq \lambda_{n}\right\}$ and $\mathbb{C}\left[\mathrm{Mat}_{n}\right]_{\lambda}$ is a simple finite dimensional $K$-module with the highest weight

$$
\left(\lambda_{1}-\lambda_{2}, \ldots, \lambda_{n-1}-\lambda_{n}, 2 \lambda_{n}, \lambda_{n-1}-\lambda_{n}, \ldots, \lambda_{1}-\lambda_{2}\right) .
$$

Similarly, $\mathrm{D}\left[\mathrm{Mat}_{n}\right]=\bigoplus_{\lambda \in \Lambda_{n}} \mathrm{D}_{\lambda}$, with $\mathrm{D}_{\lambda} \cong \mathbb{C}\left[\mathrm{Mat}_{n}\right]_{\lambda}^{*}$ (we are using here the standard pairing between polynomials and differential operators with constant coefficients).

Let $y_{\nu}=\sum_{i} v_{i} w_{i} \in \operatorname{PD}\left[\mathrm{Mat}_{n}\right]$, where $\left\{v_{i}\right\}$ is a basis in $\mathbb{C}\left[\mathrm{Mat}_{n}\right]_{\nu}$, and $\left\{w_{i}\right\}$ is the dual basis in $\left.\mathrm{D}_{\nu} \cdot y_{\nu}\right|_{\mathbb{C}\left[\mathrm{Mat}_{n}\right]_{\lambda}}$ is a scalar operator since $\mathbb{C}\left[\mathrm{Mat}_{n}\right]_{\lambda}$ is a simple $K$-module and $y_{\nu}$ is $K$-invariant. Sahi arranges studying an explicit formula for these scalars [11], [5, Proposition 3.3]:

$$
\left.\mathrm{y}_{\nu}\right|_{\mathbb{C}\left[\mathrm{Mat}_{n}\right]_{\lambda}}=\mathfrak{s}_{\nu}\left(\lambda_{1}+n-1, \lambda_{2}+n-2, \ldots, \lambda_{n-1}+1, \lambda_{n}\right),
$$

where the factorial Schur polynomial $\mathfrak{s}_{\nu}$ associated to a partition $\nu=\left(\nu_{1}, \ldots, \nu_{n}\right)$ is defined by

$$
\mathfrak{s}_{\nu}\left(x_{1}, x_{2}, \ldots, x_{n}\right)=\frac{\operatorname{det}\left(\prod_{m=0}^{\nu_{j}+n-j-1}\left(x_{i}-m\right)\right)_{1 \leq i, j \leq n}}{\prod_{i<j}\left(x_{i}-x_{j}\right)}
$$

(see [2, 9]).

This paper presents a $q$-analog of this formula. 


\section{The main statement}

Let $q \in(0,1)$. All algebras are assumed to be associative and unital, and $\mathbb{C}$ is the ground field.

Recall that $U_{q} \mathfrak{s l}_{2 n}$ is a Hopf algebra with generators $\left\{E_{i}, F_{i}, K_{i}, K_{i}^{-1}\right\}_{i=1}^{2 n-1}$ and the DrinfeldJimbo relations 3 ]

$$
\begin{gathered}
K_{i} K_{j}=K_{j} K_{i}, \quad K_{i} K_{i}^{-1}=K_{i}^{-1} K_{i}=1 ; \\
K_{i} E_{i}=q^{2} E_{i} K_{i}, \quad K_{i} F_{i}=q^{-2} F_{i} K_{i} ; \\
K_{i} E_{j}=q^{-1} E_{j} K_{i}, \quad K_{i} F_{j}=q F_{j} K_{i}, \quad|i-j|=1 ; \\
K_{i} E_{j}=E_{j} K_{i}, \quad K_{i} F_{j}=F_{j} K_{i}, \quad|i-j|>1 ; \\
E_{i} F_{j}-F_{j} E_{i}=\delta_{i j} \frac{K_{i}-K_{i}^{-1}}{q-q^{-1}} ; \\
E_{i}^{2} E_{j}-\left(q+q^{-1}\right) E_{i} E_{j} E_{i}+E_{j} E_{i}^{2}=0, \quad|i-j|=1 ; \\
F_{i}^{2} F_{j}-\left(q+q^{-1}\right) F_{i} F_{j} F_{i}+F_{j} F_{i}^{2}=0, \quad|i-j|=1 ; \\
E_{i} E_{j}-E_{j} E_{i}=F_{i} F_{j}-F_{j} F_{i}=0, \quad|i-j| \neq 1 .
\end{gathered}
$$

The coproduct, the counit, and the antipode are defined as follows:

$$
\begin{aligned}
& \triangle E_{j}=E_{j} \otimes 1+K_{j} \otimes E_{j}, \quad \varepsilon\left(E_{j}\right)=0, \quad S\left(E_{j}\right)=-K_{j}^{-1} E_{j}, \\
& \triangle F_{j}=F_{j} \otimes K_{j}^{-1}+1 \otimes F_{j}, \quad \varepsilon\left(F_{j}\right)=0, \quad S\left(F_{j}\right)=-F_{j} K_{j}, \\
& \triangle K_{j}=K_{j} \otimes K_{j}, \quad \varepsilon\left(K_{j}\right)=1, \quad S\left(K_{j}\right)=K_{j}^{-1}, \quad j=1, \ldots, 2 n-1 .
\end{aligned}
$$

Equip the Hopf algebra $U_{q} \mathfrak{s l}_{2 n}$ with an involution $*$ :

$$
\left(K_{j}^{ \pm 1}\right)^{*}=K_{j}^{ \pm 1}, \quad E_{j}^{*}=\left\{\begin{array}{rl}
K_{j} F_{j}, & j \neq n, \\
-K_{j} F_{j}, & j=n,
\end{array} \quad F_{j}^{*}=\left\{\begin{array}{cc}
E_{j} K_{j}^{-1}, & j \neq n \\
-E_{j} K_{j}^{-1}, & j=n .
\end{array}\right.\right.
$$

$U_{q} \mathfrak{s u}_{n, n} \stackrel{\text { def }}{=}\left(U_{q} \mathfrak{s l}_{2 n}, *\right)$ is a $*$-Hopf algebra. Denote by $U_{q} \mathfrak{k} \subset U_{q} \mathfrak{s l}_{2 n}$ the Hopf subalgebra generated by $E_{j}, F_{j}, j \neq n$, and $K_{i}, K_{i}^{-1}, i=1, \ldots, 2 n-1$.

Introduce a $*$-algebra $\operatorname{Pol}\left(\mathrm{Mat}_{n}\right)_{q}$, which is one of the basic objects in the theory of quantum bounded symmetric domains (see, for example, [13]). First, introduce a well-known quantum analog $\mathbb{C}\left[\mathrm{Mat}_{n}\right]_{q}$ of the algebra $\mathbb{C}\left[\mathrm{Mat}_{n}\right]$ of holomorphic polynomials on the matrix space (see, for example, 4], chap. 9.2). It is defined by the generators $z_{a}^{\alpha}, a, \alpha=1, \ldots, n$, and the following relations

$$
\begin{array}{llll}
z_{a}^{\alpha} z_{b}^{\beta}-q z_{b}^{\beta} z_{a}^{\alpha}=0, & a=b \quad \& \quad \alpha<\beta, \quad \text { or } \quad a<b \quad \& \quad \alpha=\beta, \\
z_{a}^{\alpha} z_{b}^{\beta}-z_{b}^{\beta} z_{a}^{\alpha}=0, & \alpha<\beta \quad \& \quad a>b, \\
z_{a}^{\alpha} z_{b}^{\beta}-z_{b}^{\beta} z_{a}^{\alpha}-\left(q-q^{-1}\right) z_{a}^{\beta} z_{b}^{\alpha}=0, & \alpha<\beta \quad \& \quad a<b .
\end{array}
$$

Similarly, denote by $\mathbb{C}\left[\overline{\mathrm{Mat}}_{n}\right]_{q}$ an algebra with the generators $\left(z_{a}^{\alpha}\right)^{*}, a, \alpha=1, \ldots, n$ and the defining relations

$$
\begin{array}{ll}
\left(z_{b}^{\beta}\right)^{*}\left(z_{a}^{\alpha}\right)^{*}-q\left(z_{a}^{\alpha}\right)^{*}\left(z_{b}^{\beta}\right)^{*}=0, & a=b \& \alpha<\beta, \quad \text { or } \quad a<b \& \alpha=\beta, \\
\left(z_{b}^{\beta}\right)^{*}\left(z_{a}^{\alpha}\right)^{*}-\left(z_{a}^{\alpha}\right)^{*}\left(z_{b}^{\beta}\right)^{*}=0, & \alpha<\beta \& a>b, \\
\left(z_{b}^{\beta}\right)^{*}\left(z_{a}^{\alpha}\right)^{*}-\left(z_{a}^{\alpha}\right)^{*}\left(z_{b}^{\beta}\right)^{*}-\left(q-q^{-1}\right)\left(z_{b}^{\alpha}\right)^{*}\left(z_{a}^{\beta}\right)^{*}=0, & \alpha<\beta \& a<b .
\end{array}
$$


Let $\mathbb{C}\left[\mathrm{Mat}_{n} \oplus \overline{\mathrm{Mat}}_{n}\right]_{q}$ be an algebra with the generators $z_{a}^{\alpha},\left(z_{a}^{\alpha}\right)^{*}, a, \alpha=1, \ldots, n$, and the defining relations (3) - (8) and

$$
\left(z_{b}^{\beta}\right)^{*} z_{a}^{\alpha}=q^{2} \sum_{a^{\prime}, b^{\prime}=1}^{n} \sum_{\alpha^{\prime}, \beta^{\prime}=1}^{n} R\left(b, a, b^{\prime}, a^{\prime}\right) R\left(\beta, \alpha, \beta^{\prime}, \alpha^{\prime}\right) z_{a^{\prime}}^{\alpha^{\prime}}\left(z_{b^{\prime}}^{\beta^{\prime}}\right)^{*}+\left(1-q^{2}\right) \delta_{a b} \delta^{\alpha \beta},
$$

where $\delta_{a b}, \delta^{\alpha \beta}$ are Kronecker symbols,

$$
R\left(j, i, j^{\prime}, i^{\prime}\right)=\left\{\begin{array}{cl}
q^{-1}, & i \neq j \& j=j^{\prime} \& i=i^{\prime}, \\
1, & i=j=i^{\prime}=j^{\prime}, \\
-\left(q^{-2}-1\right), & i=j \& i^{\prime}=j^{\prime} \& i^{\prime}>i, \\
0, & \text { otherwise. }
\end{array}\right.
$$

Finally, let $\operatorname{Pol}\left(\mathrm{Mat}_{n}\right)_{q}$ denotes the $*$-algebra $\left(\mathbb{C}\left[\mathrm{Mat}_{n} \oplus \overline{\mathrm{Mat}}_{n}\right]_{q}, *\right)$ with the involution given by $*: z_{a}^{\alpha} \mapsto\left(z_{a}^{\alpha}\right)^{*}$.

It is very important for our purposes that $\operatorname{Pol}\left(\mathrm{Mat}_{n}\right)_{q}$ is a $q$-analog of the algebra of differential operators with polynomial coefficients $\mathrm{PD}\left[\mathrm{Mat}_{n}\right]$ mentioned in the Introduction. Indeed, the latter algebra is derivable from $\mathrm{Pol}\left(\mathrm{Mat}_{n}\right)_{q}$ via the change of generators $z_{a}^{\alpha} \rightarrow\left(1-q^{2}\right)^{-1 / 2} z_{a}^{\alpha}$ and a subsequent formal passage to the limit as $q \rightarrow 1$.

$\operatorname{Pol}\left[\mathrm{Mat}_{n}\right]_{q}$ can be equipped with a $U_{q} \mathfrak{s u}{ }_{n, n}$-module algebra structure via such formulas (see [13, Sec. 9,10]): for $a, \alpha=1, \ldots, n$

$$
\begin{gathered}
K_{n}^{ \pm 1} z_{a}^{\alpha}= \begin{cases}q^{ \pm 2} z_{a}^{\alpha}, & a=n \& \alpha=n, \\
q^{ \pm 1} z_{a}^{\alpha}, & a=n \& \alpha \neq n \text { or } \quad a \neq n \& \alpha=n, \\
z_{a}^{\alpha}, & \text { otherwise, }\end{cases} \\
F_{n} z_{a}^{\alpha}=q^{1 / 2} \cdot\left\{\begin{array}{ll}
1, & a=n \& \alpha=n, \\
0, & \text { otherwise, }
\end{array} \quad E_{n} z_{a}^{\alpha}=-q^{1 / 2} \cdot \begin{cases}q^{-1} z_{a}^{n} z_{n}^{\alpha}, & a \neq n \& \alpha \neq n, \\
\left(z_{n}^{n}\right)^{2}, & a=n \& \alpha=n, \\
z_{n}^{n} z_{a}^{\alpha}, & \text { otherwise, }\end{cases} \right.
\end{gathered}
$$

and for $k \neq n$

$$
\begin{aligned}
& K_{k}^{ \pm 1} z_{a}^{\alpha}= \begin{cases}q^{ \pm 1} z_{a}^{\alpha}, & k<n \& a=k \quad \text { or } \quad k>n \& \alpha=2 n-k, \\
q^{\mp 1} z_{a}^{\alpha}, & k<n \& a=k+1 \quad \text { or } \quad k>n \& \alpha=2 n-k+1, \\
z_{a}^{\alpha}, & \text { otherwise, }\end{cases} \\
& F_{k} z_{a}^{\alpha}=q^{1 / 2} \cdot \begin{cases}z_{a+1}^{\alpha}, & k<n \& a=k, \\
z_{a}^{\alpha+1}, & k>n \& \alpha=2 n-k, \\
0, & \text { otherwise, }\end{cases} \\
& E_{k} z_{a}^{\alpha}=q^{-1 / 2} \cdot \begin{cases}z_{a-1}^{\alpha}, & k<n \& a=k+1, \\
z_{a}^{\alpha-1}, & k>n \& \alpha=2 n-k+1, \\
0, & \text { otherwise. }\end{cases}
\end{aligned}
$$

In the sequel we are using standard results on finite dimensional $U_{q} \mathfrak{k}$-modules of

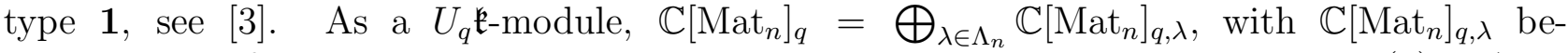
ing a simple finite dimensional $U_{q} \mathfrak{k}$-module with the highest weight given by (11). Also,

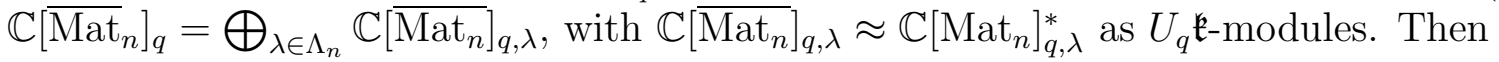

$$
\left(\operatorname{Pol}\left(\operatorname{Mat}_{n}\right)_{q}\right)^{U_{q} \mathfrak{k}}=\bigoplus_{\nu \in \Lambda_{n}} \mathrm{Pl}_{\nu}, \quad \mathrm{Pl}_{\nu}=\left(\mathbb{C}\left[\operatorname{Mat}_{n}\right]_{q, \nu} \otimes \mathbb{C}\left[\overline{\operatorname{Mat}_{n}}\right]_{q, \nu}\right)^{U_{q} \mathfrak{k}}, \quad \operatorname{dim} \mathrm{Pl}_{\nu}=1 .
$$


Let $\left\{v_{j}\right\} \subset \mathbb{C}\left[\mathrm{Mat}_{n}\right]_{q, \nu}$ be a basis and $\left\{w_{j}\right\} \subset \mathbb{C}\left[\overline{\mathrm{Mat}_{n}}\right]_{q, \nu}$ the dual basis. Then $\sum_{j} v_{j} w_{j} \in \mathrm{Pl}_{\nu}$. Introduce $q$-minors

$$
\begin{gathered}
z_{I}^{\wedge k} \stackrel{\text { def }}{=} \sum_{s \in S_{k}}(-q)^{l(s)} z_{i_{1}}^{j_{s(1)}} z_{i_{2}}^{j_{s(2)}} \cdot \ldots \cdot z_{i_{k}}^{j_{s(k)}}, \\
I=\left\{\left(i_{1}, i_{2}, \ldots, i_{k}\right) \mid 1 \leq i_{1}<i_{2}<\ldots<i_{k} \leq n\right\}, \\
J=\left\{\left(j_{1}, j_{2}, \ldots, j_{k}\right) \mid 1 \leq j_{1}<j_{2}<\ldots<j_{k} \leq n\right\} .
\end{gathered}
$$

It can be verified easily that

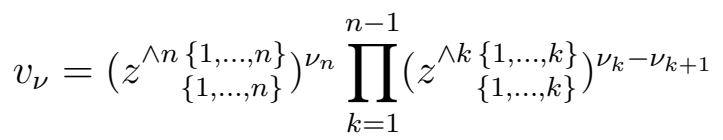

is a highest weight vector of $\mathbb{C}\left[\mathrm{Mat}_{n}\right]_{q, \nu}$. Consider a basis $\left\{v_{j}\right\} \subset \mathbb{C}\left[\mathrm{Mat}_{n}\right]_{q, \nu}$ that contains $v_{\nu}$. The isomorphism between $\mathbb{C}\left[\overline{\mathrm{Mat}}_{n}\right]_{q, \nu}$ and $\mathbb{C}\left[\mathrm{Mat}_{n}\right]_{q, \nu}^{*}$ mentioned above can be chosen so that the dual basis $\left\{w_{j}\right\}$ contains $v_{\nu}^{*}$. Introduce $y_{\nu} \in \mathrm{Pl}_{\nu}$ by

$$
y_{\nu}=\sum_{j} v_{j} w_{j}
$$

Denote by $\mathcal{H}$ a $\operatorname{Pol}\left(\mathrm{Mat}_{n}\right)_{q}$-module with a generator $f_{0}$ and defining relations

$$
\left(z_{a}^{\alpha}\right)^{*} f_{0}=0, \quad a, \alpha=1, \ldots, n,
$$

and by $T_{F}$ the corresponding representation of $\operatorname{Pol}\left(\mathrm{Mat}_{n}\right)_{q}$ in $\mathcal{H}$. The statements of the following proposition are proved in [13].

\section{Proposition 1 1. $\mathcal{H}=\mathbb{C}\left[\mathrm{Mat}_{n}\right]_{q} f_{0}$.}

2. $\mathcal{H}$ is a simple $\operatorname{Pol}\left(\mathrm{Mat}_{n}\right)_{q}$-module.

3. There exists a unique sesquilinear form $(\cdot, \cdot)$ on $\mathcal{H}$ with the following properties:

i) $\left(f_{0}, f_{0}\right)=1$; ii $)(f v, w)=\left(v, f^{*} w\right)$ for all $v, w \in \mathcal{H}, f \in \operatorname{Pol}\left(\operatorname{Mat}_{n}\right)_{q}$.

4. The form $(\cdot, \cdot)$ is positive definite.

5. $T_{F}$ is a faithful representation.

So, $\mathcal{H}$ is a pre-Hilbert space, and $T_{F}$ is an irreducible faithful $*$-representation.

Evidentally, $\mathcal{H}$ inherits the decomposition

$$
\mathcal{H}=\bigoplus_{\lambda \in \Lambda_{n}} \mathcal{H}_{\lambda}
$$

Proposition 2 (D. Shklyarov) $y_{\nu} y_{\lambda}=y_{\lambda} y_{\nu}$ for all partitions $\nu \neq \lambda$.

Proof. Commutativity is deduced from the faithfulness of $T_{F}$ and the simplicity of the summands in (10).

As in the classical case, $\left.T_{F}\left(y_{\nu}\right)\right|_{\mathcal{H}_{\lambda}}$ are scalar operators for all $\nu$ and $\lambda$. The main goal of this paper is to obtain an explicit formula for the scalars $\left.T_{F}\left(y_{\nu}\right)\right|_{\mathcal{H}_{\lambda}}$.

Recall the notation of the $q$-factorial Schur polynomials [5]: for $\nu \in \Lambda_{n}$

$$
\mathfrak{s}_{\nu}\left(x_{1}, x_{2}, \ldots, x_{n} ; q\right)=\frac{\left.\operatorname{det}\left(\prod_{m=0}^{\nu_{j}+n-j-1}\left(x_{i}-q^{m}\right)\right)\right)_{1 \leq i, j \leq n}}{\prod_{i<j}\left(x_{i}-x_{j}\right)} .
$$


Theorem 1 For all partitions $\nu, \lambda \in \Lambda_{n}$

$$
\left.T_{F}\left(y_{\nu}\right)\right|_{\mathcal{H}_{\lambda}}=(-q)^{\sum_{i=1}^{n} \nu_{i}} q^{\text {const }} \mathfrak{s}_{\nu}\left(q^{2\left(\lambda_{1}+n-1\right)}, q^{2\left(\lambda_{2}+n-2\right)}, \ldots, q^{2\left(\lambda_{n-1}+1\right)}, q^{2 \lambda_{n}} ; q^{2}\right)
$$

with const $=-\sum_{i=1}^{n} \nu_{i}\left(\nu_{i}+2 n-2 i\right)$.

This theorem is a natural generalization of the following result. Let $\mathbf{1}^{k} \stackrel{\text { def }}{=}(\underbrace{1, \ldots, 1}_{k}, 0, \ldots, 0)$.

Theorem 2 [1, Theorem 1] For all $k=1,2, \ldots, n$ and all $\lambda \in \Lambda_{n}$

$$
\left.T_{F}\left(y_{\mathbf{1}^{k}}\right)\right|_{\mathcal{H}_{\lambda}}=(-q)^{k} q^{-k^{2}-2 k(n-k)} \mathfrak{s}_{1^{k}}\left(q^{2\left(\lambda_{1}+n-1\right)}, q^{2\left(\lambda_{2}+n-2\right)}, \ldots, q^{2\left(\lambda_{n-1}+1\right)}, q^{2 \lambda_{n}} ; q^{2}\right) .
$$

First, we prove some auxiliary statements.

Lemma 1 The subalgebra $\operatorname{Pol}\left(\operatorname{Mat}_{n}\right)_{q}^{U_{q} \mathfrak{k}}$ is generated by the elements $y_{1^{k}}$.

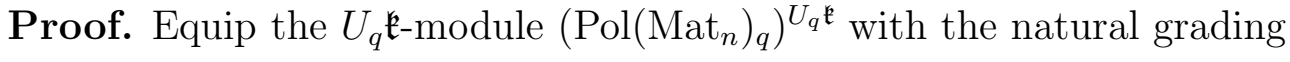

$$
\left(\operatorname{Pol}\left(\operatorname{Mat}_{n}\right)_{q}\right)^{U_{q} \mathfrak{k}}=\bigoplus_{j=0}^{\infty}\left(\operatorname{Pol}\left(\operatorname{Mat}_{n}\right)_{q}\right)_{j}^{U_{q} \mathfrak{k}}, \quad\left(\operatorname{Pol}\left(\mathrm{Mat}_{n}\right)_{q}\right)_{j}^{U_{q} \mathfrak{k}}=\bigoplus_{\nu \in \Lambda_{n},|\nu|=j} \mathrm{Pl}_{\nu}
$$

Hence $\operatorname{dim}\left(\operatorname{Pol}\left(\operatorname{Mat}_{n}\right)_{q}\right)_{j}^{U_{q} \mathfrak{k}}=\#\left\{\nu \in \Lambda_{n},|\nu|=j\right\}$.

It follows from Theorem 2 that monomials $\left\{y_{\mathbf{1}^{1}}^{a_{1}} y_{\mathbf{1}^{2}}^{a_{2} \ldots} y_{\mathbf{1}^{n}}^{a_{n}}\right\}$ are linear independent. This

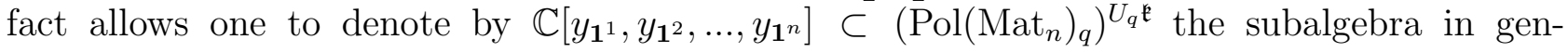
erated by the elements $y_{1^{1}}, y_{1^{2}}, \ldots, y_{1^{n}}$. It is easy to see that $\operatorname{deg} y_{1^{k}}=k$. Hence $\mathbb{C}\left[y_{1^{1}}, y_{1^{2}}, \ldots, y_{1^{n}}\right] \bigcap\left(\operatorname{Pol}\left(\operatorname{Mat}_{n}\right)_{q}\right)_{j}^{U_{q} \mathfrak{k}}$ is a linear span of $\left\{y_{1^{1}}^{a_{1}} y_{\mathbf{1}^{2}}^{a_{2}} \ldots y_{\mathbf{1}^{n}}^{a_{n}} \mid a_{1}+2 a_{2}+\ldots+n a_{n}=j\right\}$, and

$$
\operatorname{dim}\left(\mathbb{C}\left[y_{1^{1}}, y_{1^{2}}, \ldots, y_{1^{n}}\right] \bigcap\left(\operatorname{Pol}\left(\operatorname{Mat}_{n}\right)_{q}\right)_{j}^{U_{q} \mathfrak{k}}\right)=\#\left\{a_{1}, \ldots, a_{n} \in \mathbb{Z}_{+} \mid a_{1}+2 a_{2}+\ldots+n a_{n}=j\right\} .
$$

So, $\operatorname{dim}\left(\mathbb{C}\left[y_{1^{1}}, y_{1^{2}}, \ldots, y_{1^{n}}\right] \bigcap\left(\operatorname{Pol}\left(\operatorname{Mat}_{n}\right)_{q}\right)_{j}^{U_{q} \mathfrak{k}}\right)=\operatorname{dim}\left(\operatorname{Pol}\left(\operatorname{Mat}_{n}\right)_{q}\right)_{j}^{U_{q} \mathfrak{k}}$, and $\left(\operatorname{Pol}\left(\operatorname{Mat}_{n}\right)_{q}\right)^{U_{q} \mathfrak{k}}$ is generated by $y_{1^{1}}, y_{1^{2}}, \ldots, y_{1^{n}}$.

The next statements concern symmetric polynomials and vanishing conditions in the spirit of papers [6, 10] (for their classical analogs, see [5, 7, 8]). Recall some notations from [6]. Fix non-zero real numbers $q$ and $t$. For every $\lambda \in \Lambda_{n}$ we define $\bar{\lambda}=\left(q^{\lambda_{1}}, q^{\lambda_{2}} t^{-1}, \ldots, q^{\lambda_{n}} t^{-n+1}\right)$. We use the following short notation: $|\lambda|=\sum_{i=1}^{n} \lambda_{i}$ for $\lambda \in \Lambda_{n}$. Also, let $m_{\lambda}$ be the monomial symmetric polynomial that corresponds to $\lambda \in \Lambda_{n}$. Recall that the usual order on $\Lambda_{n}$ : for $\lambda, \mu \in \Lambda_{n}$ we say $\lambda \geq \mu$ if $\lambda_{1}+\ldots+\lambda_{i} \geq \mu_{1}+\ldots+\mu_{i}$ for all $i=1, \ldots, n$.

Proposition 3 [6, Theorem 2.4] For every $\lambda \in \Lambda_{n}$ there exists a unique symmetric polynomial $P_{\lambda}(z ; q, t)$ in $n$ variables such that $P_{\lambda}(\bar{\mu} ; q, t)=0$ for all $\mu \in \Lambda_{n},|\mu| \leq|\lambda|, \mu \neq \lambda$, and which has an expansion $P_{\lambda}(z ; q, t)=\sum_{\mu \leq \lambda} p_{\lambda \mu} m_{\mu}(z)$ with $p_{\lambda \lambda}=1$.

Proposition 4 [6, Proposition 2.8] $P_{\lambda}(z ; q, q)=q^{-(n-1)|\lambda|} \mathfrak{s}_{\lambda}\left(q^{n-1} z ; q\right)$.

Now we can prove

Lemma 2 For any partition $\nu$ there exists a constant $c_{\nu}$ such that for all $\lambda \in \Lambda_{n}$

$$
\left.T_{F}\left(y_{\nu}\right)\right|_{\mathcal{H}_{\lambda}}=c_{\nu} \mathfrak{s}_{\nu}\left(q^{2\left(\lambda_{1}+n-1\right)}, q^{2\left(\lambda_{2}+n-2\right)}, \ldots, q^{2\left(\lambda_{n-1}+1\right)}, q^{2 \lambda_{n}} ; q^{2}\right) .
$$


Proof. It follows from Theorem 2 and Lemma 1, that for an arbitrary partition $\nu$ there exists a symmetric polynomial of degree $|\nu|$ in $n$ variables $x_{1}, \ldots, x_{n}$, such that the eigenvalues $\left.T_{F}\left(y_{\nu}\right)\right|_{\mathcal{H}_{\lambda}}$ are just the values of the polynomial at $x_{1}=q^{2\left(\lambda_{1}+n-1\right)}, \ldots, x_{n}=q^{2 \lambda_{n}}$.

Let $\delta=(n-1, \ldots, 1,0), q^{2(\mu+\delta)} \stackrel{\text { def }}{=}\left(q^{2\left(\mu_{1}+n-1\right)}, q^{2\left(\mu_{2}+n-2\right)}, \ldots, q^{2\left(\mu_{n-1}+1\right)}, q^{2 \mu_{n}}\right)$ for any $\mu \in \Lambda_{n}$. Propositions 3 and 4 claim that $\mathfrak{s}_{\nu}\left(x_{1}, \ldots, x_{n} ; q^{2}\right)$ is a unique (up to a constant multiplier) symmetric polynomial of degree $|\nu|$ with $\mathfrak{s}_{\nu}\left(q^{2(\mu+\delta)} ; q^{2}\right)=0$ for all $\mu \in \Lambda_{n},|\mu| \leq|\nu|, \mu \neq \nu$.

To finish the proof, one should investigate zeros of $T_{F}\left(y_{\nu}\right)$ to conclude the proof (cf. the proof in Sahi's paper [1]]). We claim that

$$
\left.T_{F}\left(y_{\nu}\right)\right|_{\mathcal{H}_{\lambda}}=0 \text { for }|\nu| \leq|\lambda| \quad \text { unless } \quad \nu=\lambda \text {. }
$$

Indeed, it suffices to prove that $T_{F}\left(y_{\nu}\right)\left(v_{\lambda} f_{0}\right)=0$ for partitions $\nu$ and $\lambda$ such that $|\nu| \leq|\lambda|$, $\nu \neq \lambda$. Recall that $y_{\nu}=\sum v_{j} w_{j}$, where $\left\{v_{j}\right\} \subset \mathbb{C}\left[\mathrm{Mat}_{n}\right]_{q, \nu}$ contains $v_{\nu}$ and $\left\{w_{j}\right\} \subset \mathbb{C}\left[\overline{\mathrm{Mat}_{n}}\right]_{q, \nu}$ contains $v_{\nu}^{*}$. It follows from the commutation relations (9) that $T_{F}\left(w_{j}\right)\left(v_{\lambda} f_{0}\right)=0$ unless $w_{j}=v_{\lambda}^{*}$. So, $T_{F}\left(y_{\nu}\right)\left(v_{\lambda} f_{0}\right)=0$ unless $\nu=\lambda$.

Introduce the notations: $\lambda-a \mathbf{1}^{n}=\left(\lambda_{1}-a, \ldots, \lambda_{n}-a\right)$. The next proposition completes the proof of Theorem 1.

Proposition $5 \quad c_{\nu}=(-q)^{|\nu|} q^{-\sum_{i=1}^{n} \nu_{i}\left(\nu_{i}+2 n-2 i\right)}$.

Proof. Let us compare $T_{F}\left(y_{\nu}\right)\left(v_{\nu} f_{0}\right)$ and $\mathfrak{s}_{\nu}\left(q^{2(\nu+\delta)} ; q^{2}\right)$. By Proposition 6 ,

$$
\left.T_{F}\left(y_{\nu}\right)\left(v_{\nu} f_{0}\right)=\left(\operatorname{det}_{q} \mathbf{z}\right)^{\nu_{n}} T_{F}\left(y_{\nu-\nu_{n} \mathbf{1}^{n}}\right) T_{F}\left(\left(\operatorname{det}_{q} \mathbf{z}\right)^{*}\right)^{\nu_{n}}\right) v_{\nu} f_{0}
$$

By Theorem 2 ,

$$
\left.T_{F}\left(\left(\operatorname{det}_{q} \mathbf{z}\right)^{*}\right)^{\nu_{n}}\right) v_{\nu} f_{0}=(-1)^{n \nu_{n}} q^{-n(n-1) \nu_{n}} \prod_{i=0}^{\nu_{n}-1} \mathfrak{s}_{1^{n}}\left(q^{2\left(\nu+\delta-i \mathbf{1}^{n}\right)} ; q^{2}\right) v_{\nu-\nu_{n} \mathbf{1}^{n}} f_{0} .
$$

We proceed by induction in $n$. For $n=1$ the statement follows from the last identity and Lemma 3 from the next section.

Let $n>1$. One can rewrite some of the commutation relations (9) more explicitly:

$$
\begin{aligned}
& \left(z_{n}^{\alpha}\right)^{*} z_{b}^{\beta}=q \sum_{\alpha^{\prime}, \beta^{\prime}=1}^{n} R\left(\alpha, \beta, \alpha^{\prime}, \beta^{\prime}\right) z_{b}^{\beta^{\prime}}\left(z_{n}^{\alpha^{\prime}}\right)^{*} \quad \text { for } \quad b<n, \\
& \left(z_{a}^{n}\right)^{*} z_{b}^{\beta}=q \sum_{a^{\prime}, b^{\prime}=1}^{n} R\left(a, b, a^{\prime}, b^{\prime}\right) z_{b^{\prime}}^{\beta}\left(z_{a^{\prime}}^{n}\right)^{*} \quad \text { for } \quad \beta<n .
\end{aligned}
$$

Hence, $T_{F}\left(\left(z_{a}^{\alpha}\right)^{*}\right) v_{\nu-\nu_{n} \mathbf{1}^{n}} f_{0}=0$ for $a=n$ or $\alpha=n$. Denote by $T_{F}^{\prime}$ the faithful representation of $\operatorname{Pol}\left(\mathrm{Mat}_{n-1}\right)_{q}$ in the vector space $\mathcal{H}^{\prime}$ defined by a single generator $f_{0}^{\prime}$ and the relations $\left(z_{a}^{\alpha}\right)^{*} f_{0}^{\prime}=0$, for $a, \alpha=1, \ldots, n-1$. Thus,

$$
\left.T_{F}\left(y_{\nu-\nu_{n} \mathbf{1}^{n}}\right)\right|_{\mathcal{H}_{\nu-\nu_{n} \mathbf{1}^{n}}}=\left.T_{F}^{\prime}\left(y_{\tau}\right)\right|_{\mathcal{H}^{\prime}{ }_{\tau}},
$$

where $\tau=\left(\nu_{1}-\nu_{n}, \ldots, \nu_{n-1}-\nu_{n}\right)$. Let $\delta^{\prime}=(n-2, \ldots, 2,1,0)$. Hence, by the inductive assumption,

$$
T_{F}\left(y_{\nu-\nu_{n} \mathbf{1}^{n}}\right) v_{\nu-\nu_{n} \mathbf{1}^{n}} f_{0}=(-q)^{|\tau|} q^{-\sum_{i=1}^{n-1} \tau_{i}\left(\tau_{i}+2 n-2-2 i\right)} \mathfrak{s}_{\tau}\left(q^{2\left(\tau+\delta^{\prime}\right)} ; q^{2}\right) v_{\nu-\nu_{n} \mathbf{1}^{n}} f_{0}
$$


Now the required statement follows from Lemmas 3, 4 of the next section and the following computation

$$
\begin{aligned}
& T_{F}\left(y_{\nu}\right) v_{\nu} f_{0}=(-1)^{n \nu_{n}} q^{-n(n-1) \nu_{n}} \prod_{i=0}^{\nu_{n}-1} \mathfrak{s}_{1^{n}}\left(q^{2\left(\nu+\delta-i \mathbf{1}^{n}\right)} ; q^{2}\right)\left(\operatorname{det}_{q} \mathbf{z}\right)^{\nu_{n}} T_{F}\left(y_{\nu-\nu_{n} \mathbf{1}^{n}}\right) v_{\nu-\nu_{n} \mathbf{1}^{n}} f_{0}= \\
& (-1)^{n \nu_{n}} q^{-n(n-1) \nu_{n}} \prod_{i=0}^{\nu_{n}-1} \mathfrak{s}_{1^{n}}\left(q^{2\left(\nu+\delta-i \mathbf{1}^{n}\right)} ; q^{2}\right)(-q)^{|\tau|} q^{-\sum_{i=1}^{n-1} \tau_{i}\left(\tau_{i}+2 n-2-2 i\right)} \mathfrak{s}_{\tau}\left(q^{2\left(\tau+\delta^{\prime}\right)} ; q^{2}\right) v_{\nu} f_{0}= \\
& (-q)^{|\nu|} q^{-\sum_{i=1}^{n} \nu_{i}\left(\nu_{i}+2 n-2 i\right)} \mathfrak{s}_{\nu}\left(q^{2(\nu+\delta)} ; q^{2}\right) v_{\nu} f_{0} .
\end{aligned}
$$

Proposition $6 y_{\nu}=\left(\operatorname{det}_{q} \mathbf{z}\right)^{\nu_{n}} y_{\nu-\nu_{n} \mathbf{1}^{n}}\left(\left(\operatorname{det}_{q} \mathbf{z}\right)^{*}\right)^{\nu_{n}}$.

Proof. It is obvious that $\left(\operatorname{det}_{q} \mathbf{z}\right)^{\nu_{n}} y_{\nu-\nu_{n} \mathbf{1}^{n}}\left(\left(\operatorname{det}_{q} \mathbf{z}\right)^{*}\right)^{\nu_{n}} \in \mathrm{Pl}_{\nu}=\mathbb{C} \cdot y_{\nu}$, the statement follows from an explicit computation of the coefficient of $v_{\nu} v_{\nu}^{*}$.

\section{3 q-factorial Schur functions}

This section contains auxiliary statements which we used above. As usual, $(a)_{n} \stackrel{\text { def }}{=} \prod_{i=0}^{n-1}\left(a-q^{2 i}\right)$.

Lemma 3 For any partition $\nu \in \Lambda_{n}$, such that $\nu_{n}>0$, one has

$$
\mathfrak{s}_{\nu}\left(q^{2 \nu+2 \delta} ; q^{2}\right)=q^{2|\nu|-2 n} \mathfrak{s}_{1^{n}}\left(q^{2(\nu+\delta)} ; q^{2}\right) \mathfrak{s}_{\nu-\mathbf{1}^{n}}\left(q^{2\left(\nu+\delta-\mathbf{1}^{n}\right)} ; q^{2}\right)
$$

Proof. The proof reduces to the explicit computation $\mathfrak{s}_{\nu}\left(q^{2 \nu+2 \delta} ; q^{2}\right)=$

$$
\begin{aligned}
& =\prod_{1 \leq i \leq j \leq n} \frac{1}{q^{2 \nu_{i}+2 n-2 i}-q^{2 \nu_{j}+2 n-2 j}}\left|\begin{array}{cccc}
\left(q^{2 \nu_{1}+2 n-2}\right)_{\nu_{1}+n-1} & 0 & \ldots & 0 \\
\left(q^{2 \nu_{1}+2 n-2}\right)_{\nu_{2}+n-2} & \left(q^{2 \nu_{2}+2 n-4}\right)_{\nu_{2}+n-2} & \ldots & 0 \\
\ldots & \ldots & \ldots & \ldots \\
\left(q^{2 \nu_{1}+2 n-2}\right)_{\nu_{n}} & \left(q^{2 \nu_{2}+2 n-4}\right)_{\nu_{n}} & \ldots & \left(q^{2 \nu_{n}}\right)_{\nu_{n}}
\end{array}\right|= \\
& =\prod_{i=1}^{n}\left(q^{2 \nu_{i}+2 n-2 i}-1\right) q^{2 \nu_{i}+2 n-4 i-2} \prod_{1 \leq i \leq j \leq n} \frac{1}{q^{2 \nu_{i}+2 n-2 i-2}-q^{2 \nu_{j}+2 n-2 j-2}} \text {. } \\
& \cdot\left|\begin{array}{cccc}
\left(q^{2 \nu_{1}+2 n-4}\right)_{\nu_{1}+n-2} & 0 & \ldots & 0 \\
\left(q^{2 \nu_{1}+2 n-4}\right)_{\nu_{2}+n-3} & \left(q^{2 \nu_{2}+2 n-6}\right)_{\nu_{2}+n-3} & \ldots & 0 \\
\ldots & \ldots & \ldots & \ldots \\
\left(q^{2 \nu_{1}+2 n-4}\right)_{\nu_{n}-1} & \left(q^{2 \nu_{2}+2 n-6}\right)_{\nu_{n}-1} & \ldots & \left(q^{2 \nu_{n}-2}\right)_{\nu_{n}-1}
\end{array}\right| \\
& =q^{2|\nu|} q^{-2 n} \mathfrak{s}_{1^{n}}\left(q^{2 \nu+2 \delta} ; q^{2}\right) \mathfrak{s}_{\nu-\mathbf{1}^{n}}\left(q^{2\left(\nu+\delta-\mathbf{1}^{n}\right)} ; q^{2}\right)
\end{aligned}
$$

Lemma 4 For a partition $\nu \in \Lambda_{n}$ with $\nu_{n}=0$ one has $\mathfrak{s}_{\nu}\left(q^{2(\nu+\delta)} ; q^{2}\right)=q^{2|\nu|} \mathfrak{s}_{\nu^{\prime}}\left(q^{2\left(\nu^{\prime}+\delta^{\prime}\right)} ; q^{2}\right)$, where $\nu^{\prime}=\left(\nu_{1}, \ldots, \nu_{n-1}\right)$. 
Proof. The proof is managed by the explicit computation

$$
\begin{aligned}
& \mathfrak{s}_{\nu}\left(q^{2(\nu+\delta)} ; q^{2}\right)=\prod_{1 \leq i \leq j \leq n}\left(q^{2 \nu_{i}+2 n-2 i}-q^{2 \nu_{j}+2 n-2 j}\right)^{-1}\left|\begin{array}{cccc}
\left(q^{2 \nu_{1}+2 n-2}\right)_{\nu_{1}+n-1} & 0 & \ldots & 0 \\
\left(q^{2 \nu_{1}+2 n-2}\right)_{\nu_{2}+n-2} & \left(q^{2 \nu_{2}+2 n-4}\right)_{\nu_{2}+n-2} & \ldots & 0 \\
\ldots & \ldots & \ldots & \ldots \\
1 & 1 & \ldots & 1
\end{array}\right| \\
& =\prod_{1 \leq i \leq j<n}\left(q^{2 \nu_{i}+2 n-2 i}-q^{2 \nu_{j}+2 n-2 j}\right)^{-1}\left|\begin{array}{cccc}
\frac{\left(q^{2 \nu_{1}+2 n-2}\right)_{\nu_{1}+n-1}}{q^{2 \nu_{1}+2 n-2}-1} & 0 & \ldots & 0 \\
\frac{\left(q^{2 \nu_{1}+2 n-2}\right) \nu_{2}+n-2}{q^{2 \nu_{1}+2 n-2}-1} & \frac{\left(q^{2 \nu_{2}+2 n-4}\right)}{q^{2 \nu_{2}+2 n-4}-1} & \ldots & 0 \\
\ldots & \ldots & \ldots & \ldots \\
\frac{\left(q^{2 \nu_{1}+2 n-2}\right)_{\nu_{n-1}}}{q^{2 \nu_{1}+2 n-2}-1} & \frac{\left(q^{2 \nu_{2}+2 n-4}\right)_{\nu_{n-1}}}{q^{2 \nu_{2}+2 n-4}-1} & \ldots & \frac{\left(q^{2 \nu_{n-1}+2}\right)_{\nu_{n-1}}}{q^{2 \nu_{n-1}+2}-1}
\end{array}\right| \\
& =q^{2|\nu|} \mathfrak{s}_{\nu^{\prime}}\left(q^{2 \nu^{\prime}+2 \delta^{\prime}} ; q^{2}\right) .
\end{aligned}
$$

\section{Acknowledgements}

The author thanks to L. Vaksman for helping with the proof of Lemma 2 and constant attention to her work. Also thanks are due to D.Shklyarov. At last, the author would like to express her gratitude to a referee for many useful remarks.

\section{References}

[1] O. Bershtein, Ye. Kolisnyk, L. Vaksman, On a q-analog of the Wallach-Okounkov formula, - Lett. in Math.Phys. 78 No. 1 (2006), pp.97-109.

[2] L.Biedenharn, J.Louck, A new class of symmetric polynomials defined in terms of tableaux, - Adv. in Appl. Math. 10 (1989), pp.396-438.

[3] J.C. Jantzen, Lectures on Quantum Groups - Amer. Math. Soc., Providence RI, (1996).

[4] A. Klimyk, K. Schmüdgen, Quantum Groups and Their Representations - Springer, Berlin, (1997).

[5] F. Knop, S. Sahi, Difference equations and symmetric polynomials defined by their zeros, - IMRN 10 (2000), pp.473-486.

[6] F. Knop, Symmetric and non-symmetric quantum Capelli polynomials, - Comment. Math. Helv. 72 (1997), pp.84-100.

[7] B.Kostant, S.Sahi, The Capelli identity, tube domains and the generalized Laplace transform, - Adv.Math. 87 (1991), pp.71-92.

[8] B.Kostant, S.Sahi, Jordan algebras and Capelli identities, - Inv.Math. 112 (1993), pp.657664.

[9] I. Macdonald, Schur functions: theme and variations, - I.R.M.A. Strasbourg (1992) 498/S27, pp.5-39.

[10] S.Sahi, Interpolation, integrality and a generalization of Macdonald's polynomials, Int.Math.Res.Not. 10 (1996), pp.457-471. 
[11] S. Sahi, The Spectrum of certain invariant differential operators associated to a Hermitian symmetric space, - in Lie Theory and Geometry, ed. J.-L.Brylinsky, R.Brylinsky, V.Guillemin, V.Kac (1994), pp. 569-576.

[12] D. Shklyarov, S. Sinel'shchikov, L. Vaksman, q-Analogs of some bounded symmetric domains, - Czech. J. of Phys. 50 No.1 (2000), pp. 175-180.

[13] D. Shklyarov, S. Sinel'shchikov, L. Vaksman, Fock representations and quantum matrices, - International J.Math 15 No.9 (2000), pp.1-40. 